\title{
Exercise Induced Laryngeal Obstruction in a Collegiate Runner: A Case Report of a Novel Therapy
}

Cody Inskeep, MS, LAT, ATC' ; Todd Olin, MD, MSCS ${ }^{2}$; Robert Arthur MS, LAT, ATC, CSCS ${ }^{3}$

'Biotix Health, Centennial, CO; ${ }^{2}$ Pediatric Exercise Tolerance Center, Denver, CO; ${ }^{3}$ Indiana State University, Terre Haute, IN

\begin{abstract}
A 23-year-old male Division I distance runner presented with several years of exertional stridor (high-pitched inspiratory noise caused by airflow obstruction), previously diagnosed as exercised-induced laryngeal obstruction (EILO), a condition formerly known as vocal cord dysfunction and exercisedinduced paradoxical vocal fold motion. Over the course of roughly 3 years, the patient had previously failed conventional and invasive respiratory therapies for EILO including respiratory retraining, inspiratory muscle training, reflux suppression, allergy suppression, amitriptyline, performance psychology, and injection of botulinum toxin to the larynx. At a referral center that specializes in the treatment of EILO, the patient's diagnosis was confirmed through the use of a new procedure called continuous laryngoscopy during exercise. He underwent three sessions of therapeutic laryngoscopy during exercise (which relies on real-time laryngoscopy footage as biofeedback during exercise) and concurrently learned novel breathing techniques to address the problem. It is common to misdiagnose exercise induced respiratory problems based on patients-described symptoms alone. Athletic trainers should be able to recognize EILO cases and feel comfortable contacting and collaborating with expert providers on appropriate treatment. This case is also important in that it documents a treatment failure of laryngeal injection of botulinum toxin for EILO, something not previously reported in the literature.
\end{abstract}

Key Phrases

Exercise induced laryngeal obstruction, vocal cord dysfunction, respiratory

Correspondence

Cody Inskeep, Briotix Health, 9000 E Nichols Ave \#104, Centennial, CO 80112

E-mail: cody.inskeep@briotix.com

Twitter: @Codyl85

\section{Full Citation}

Inskeep C, Olin T, Arthur R. Exercise induced laryngeal obstruction in a collegiate runner: A case report of a novel therapy. Clin Pract Athl Train. 2019;2(2):37-44.

https://doi.org/10.31622/2019/0002.5.1.

Submitted: March 3, 2019 Accepted: June 10, 2019

Copyright (C) by Indiana State University All rights reserved. ISSN Online 2577-8188

\section{INTRODUCTION}

$E_{x}$ xercise-induced shortness of breath is common

in athletes, with up to $70 \%$ of athletes reporting a degree of respiratory distress during exercise. ${ }^{1,2}$ While asthma, a disease of the small airways, is the most commonly-identified respiratory disease among athletes, between 5\% and $8 \%$ of all adolescents and young adults struggle because they are experiencing airway obstruction at the level of the larynx (Video 1), a condition different than asthma. ${ }^{3}$ This upper airway obstruction is known as exercise-induced laryngeal obstruction (EILO), a condition previously referred to as vocal cord dysfunction and paradoxical vocal fold motion. It is characterized by airway obstruction that occurs at a glottic or subglottic level only during exercise (Video 2), often causing shortness of breath that can be visualized by athletic trainers.4,5 Among athletes, EILO has been often misdiagnosed as asthma, whereby in a cohort of 91 athletes referred for asthma symptoms, 31 $(35.2 \%)$ were actually diagnosed with EILO. ${ }^{5}$

At presentation, EILO symptoms often mimic those of exercise-induced asthma (EIA) with patients commonly complaining of "wheezing" in association with their dyspnea (despite the field observations that they are experiencing inspiratory stridor).6,7 EILO may be clinically distinguished from EIA by the time course of symptoms. EILO generally is characterized by symptoms that are isolated to high intensity exercise and rapidly resolve. ${ }^{8}$ In contrast, EIA generally develops over several minutes and requires up to an hour to resolve. ${ }^{9}$ In clinical settings, the physical examination of patients with 
EILO and EIA are generally normal. 10 The similarities between asthma and EILO and the common practice of relying solely on clinical history to differentiate the conditions may lead to misdiagnosis, inappropriate management, and ineffective interventions. ${ }^{7}$ However, identification of ineffective therapeutic trials with bronchodilators such as albuterol (almost universally used in the treatment of EIA) can be helpful in directing athletic trainers and other healthcare providers towards alternative diagnoses such as EILO.

The mechanisms of EILO are still unclear and current models hypothesize a variety of possible disease contributors. These factors may include laryngeal mechanical insufficiency, neural dysfunction, and a psychological component. ${ }^{7}$ Continuous laryngoscopy during exercise (Video 3), a procedure which features endoscopic visualization of the larynx throughout an entire bout of intense exercise, is currently the gold standard for diagnosis of EILO because characteristic upper airway obstruction can be visualized while field symptoms are reproduced in the exercise lab. ${ }^{11-13}$ Other methods to identify the condition indirectly including post-exercise laryngoscopy and exercise flow volume loop analysis are used in practice, but present serious limitations to EILO identification. ${ }^{10}$

Standard treatment algorithms do not currently exist for EILO although a number of therapies are used in practice. ${ }^{10}$ Removal of irritants and triggers from the athlete's environment, speech therapy, psychological interventions, pharmacological interventions, botulinum toxin injections, supraglottic surgery, and inspiratory muscle training have mixed results with no single treatment or combination of treatments showing consistent effectiveness. ${ }^{10}$ The most common treatments appear to be traditional breathing techniques taught in speech therapy. However, traditional breathing techniques often do not provide relief from exertional dyspnea and are often difficult to perform during high-intensity exercise. ${ }^{10}$ Therapeutic laryngoscopy during exercise (TLE) is a newly developed procedure that features laryngeal visualization during respiratory retraining that occurs simultaneous to exercise. ${ }^{12}$ It has also been found that after the application of traditional breathing techniques, inappropriate glottic adduction still exists during therapeutic laryngoscopy (TLE) treatments. ${ }^{14}$ In response to the observation of inconsistent success with traditional therapies and lack of treatment protocols, novel and alternative treatment techniques should be explored.

The EILO biphasic inspiration (EILOBI) techniques represent such a novel and alternative treatment. They were developed through observations made during TLE and designed for use in highintensity exercise. ${ }^{15}$ The EILOBI techniques help trigger laryngeal abduction which reduces the degree of upper respiratory obstruction in athletes with EILO. ${ }^{16}$ The following case describes a patient who was unresponsive to many common treatments for EILO, including Botulinum toxin injections, and found the greatest symptom relief with the EILOBI technique This case is important because relief from traditional therapies may not yield the most relief and alternative methods provide better results. Consulting a specialist is the best avenue for patient care.

\section{Patient Information}

\section{A 23-year-old male NCAA Division I distance} runner (Cross-Country, 3k steeplechase, 5k) with a history of childhood asthma, allergic rhinitis, and supraventricular tachycardia status post ablation presented to the healthcare team (Primary Care Physician, Immunologist, Performance Psychologist, Ear Nose and Throat Specialist, Pulmonologist) with several years of exertional dyspnea. Several months after an initial diagnosis of and treatment for asthma, he continued to experience exertional dyspnea. In the context of characterizing the dyspnea as stridor and the response to treatment as minimal, he was diagnosed with EILO. In addition to EILO, a few potential disease contributors were identified. He was found to have 
gastroesophageal reflux (GERD) and was treated with acid suppression medication and an inlet patch procedure. He was also found to struggle with anxiety and was treated by a local performance psychologist. Despite appropriate treatment of hypothesized disease contributors (allergic rhinitis, gastroesophageal reflux, and anxiety) and conventional treatment for EILO (respiratory retraining) with speech and language pathologists, he continued to struggle with exertional stridor for several months. For this reason, he was also treated with inspiratory muscle training with a POWERbreathe $\AA_{\text {, }}$ amitriptyline, and laryngeal botulism toxin injections on 3 occasions. A POWERbreathe ${ }^{\circledR}$ is an Inspiratory Muscle Training device used for improving the strength and endurance of the muscles used to breathe in; amitriptyline is a prescription drug used to treat depression, anxiety, and nerve pathologies; and botulism toxin injections are used to reduce excess muscle activity caused by dystonia. Despite these interventions which were sequentially introduced over several months, the patient continued to struggle with the primary symptom of exertional stridor through the time of his presentation at the final referral center roughly 5 years after symptoms began.

\section{Activity and Participation}

Patient reported occasional "wheezing" while running which progressed into full breathing attacks during his junior and senior years of high school that reduced his ability to run during races to a jog. Over time, these symptoms increased in terms of frequency and severity. When symptom free, which often occurred during practice, he was able to complete a mile in slightly more than 4 minutes. When symptoms occurred, he was often unable to complete races.

\section{Differential Diagnosis and Evaluation}

The differential diagnosis of exertional stridor includes all causes of fixed and dynamic intrinsic and extrinsic obstruction of the upper airway.
EILO occurring at a glottic or supraglottic level is the most likely lesion. Additionally, laryngeal webs, subglottic and tracheal stenosis, and compression from large vessels or masses can cause stridor. Prior to presentation at the Pulmonologist computed tomography of the chest and swallow evaluations excluded extrinsic compression. Previous exercise testing excluded exertional hypoxemia. Continuous laryngoscopy during exercise at the final referral center confirmed the diagnosis of EILO affecting both glottic and supraglottic structures.

\section{Body Structure and Function}

The patient was usually asymptomatic at the time of evaluation by athletic trainers in the clinic. The patient described periodic symptoms commonly associated with EIA or EILO, mainly "wheezing" with dyspnea. The few times athletic trainers were able to evaluate the patient during an episode, he presented with stridor, difficulty breathing, and altered voice pitch. These symptoms subsided within minutes of ceasing activity.

\section{Environmental and Personal Factors}

The patient comes from an affluent, supportive family who encouraged and modeled being physically active. He had goals to serve in a special-forces unit since he was a child. The student-athlete has a history of seeking treatment for anxiety that was relatively successful.

\section{INTERVENTIONS}

After diagnosis confirmation with continuous laryngoscopy during exercise, the patient performed three sessions of therapeutic laryngoscopy during exercise (TLE), described below. During the sessions, the patient learned one version of the EILOBI (EILO biphasic inspiratory) breathing techniques as well as a framework for managing some of the cognitive behavioral contributors to EILO. 


\section{OUTCOMES}

Although intermittent results were noticed with traditional respiratory therapy interventions, the patient noticed significant improvements after the series of therapeutic laryngoscopy during exercise sessions and with the EILOBI techniques. $\mathrm{He}$ routinely uses them during all of his runs. He's no longer a collegiate athlete but still averages 50 miles per week. The patient still has symptoms albeit they're less frequent and severe from a respiratory perspective if his GERD is controlled but has periods of respiratory distress. He does note, however, that if he misses a day of GERD medication his breathing while running is difficult for reasons related to pain.

\section{DISCUSSION}

We present a case of an elite male athlete with EILO who struggled with delays in diagnosis and poor responsiveness to conventional and invasive therapies for EILO including traditional respiratory retraining with trigger suppression and Botulinum toxin injections to the larynx.

This is an important case because it highlights that the EILOBI breathing techniques can result in successful treatment of EILO in elite athletes previously unresponsive to therapy. Secondarily, although previous literature seems to suggest a very high success with botulinum toxin injections, this report underscores a concern for treatment failures. ${ }^{17}$

This patient made major improvements with the use of the TLE procedure. In this procedure, patients are able to visualize their upper airway during intense exercise, with the ability to use the images as biofeedback during respiratory retraining teaching sessions (Figure 1). Additionally, the procedure enables thoughtful discussion about cognitive behavioral features which may be suspected in the event that EILO episodes trigger at somewhat unusual times. The procedure naturally enables complex teaching necessary to learn the EILOBI breathing

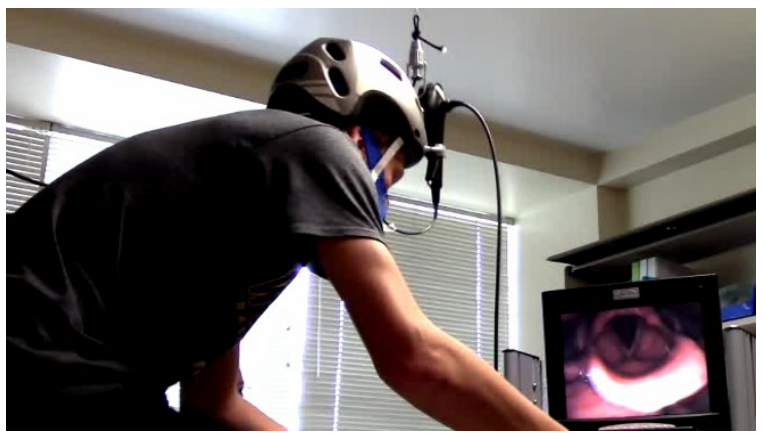

techniques. 8

Figrure 1: Therapuetic Laryngoscopy During Exercise.

The EILOBI breathing techniques feature a high resistance and low resistance phase of inspiration that can be performed rapidly. ${ }^{15}$ The techniques were discovered fortuitously during the endoscopic evaluation of patients who had previously not responded to conventionally used breathing techniques. Many of the conventional respiratory retraining strategies focus on slow breathing in the use of the diaphragm- concepts not compatible with the high respiratory rates and engagement of abdominal muscles that accompany intense exercise with athletes. ${ }^{15}$ The techniques are challenging yet important to learn and require a thoughtful teaching strategy. Nonetheless, in appropriately selected patients, like ours, that demonstrated a high degree of motor coordination, the techniques can minimize or eliminate symptoms.

\section{CLINICAL BOTTOM LINE}

It is common to diagnose and treat exerciseinduced respiratory problems based on patientsdescribed symptoms alone which may lead to misdiagnosis as asthma with subsequent inappropriate management, and ineffective interventions. Athletic trainers have a very unique perspective because they may be present during events with the opportunity to visually distinguish inspiratory stridor from other respiratory phenomena. Observation of characteristic field events or videos of characteristic episodes can 
be helpful in terms of raising suspicion of EILO. Continuous laryngoscopy during exercise can provide definitive diagnosis of EILO. Traditional breathing techniques learned from speech therapists often do not provide relief from exertional dyspnea and are difficult to perform during high-intensity exercise. The EILOBI breathing techniques were designed specifically to address these concerns. After this case presentation, athletic trainers should be able to recognize their very unique perspective on events that can occur in the field, clinically suspect EILO cases based on inspiratory stridor, and feel comfortable contacting and collaborating with expert providers on appropriate treatment.

\section{PATIENT PERSPECTIVE}

My breathing problems began during my sophomore year of high school. I had been a tennis player who occasionally ran until I took up running full time during my freshman year of high school where I was able to make it to the state regional meet. I had been a diagnosed asthmatic since I was 8 years old and occasionally used an inhaler during wheezing episodes. Despite wheezing at times, I was still a competitive tennis player who was ranked in the top 5 in the state.

At the conclusion of my freshman season we wanted more answers so I saw an immunologist. I tested positive for multiple allergens. We were excited for this news as we thought we had found the reason for my breathing issues that were starting to develop. Initially I had thought my issues were from pre-race anxiety but the confirmation of having multiple allergies seemed like a more likely cause. I started getting routine allergy shots which led to a sophomore track season where I had very few breathing issues.

My junior and senior year of high school were drastically different. I routinely had significant breathing issues during multi-race days, some of which would reduce my pace to a jog with bad wheezing. There was no consistency to causes to

Copyright $(\subseteq$ Indiana State University ISSN Online 2577-8188 these issues; high- and low-pressure races, time of year, nothing was consistent. During this time my race anxiety seemed to escalate and I would sometimes puke before or after races. By this time, I had been to the doctor multiple times and had been told I was anemic, had asthma, and was over training.

During my freshman year of college in 2013,1 had very few breathing attacks early in the season; however, when I did have them they were severe and the severity seemed to be getting worse. As the season progressed the breathing attacks became more frequent, which was a pattern for my entire college career. I can only think of one season where the first real workout of the year didn't go amazing. I chalked this pattern up to being less stressed coming out of the summer but when I thought about my anxiety levels before a fast workout, there didn't seem to be any correlation. It seemed as though the more fit I got through the season, the worse and more frequent my attacks would become.

By the spring season during my freshman year I was able to run workouts in practice very well but struggled during competition. From the outside it appeared as if I was overtraining and couldn't handle the pressure while racing. I don't have any hard feelings for anybody who thought that because it seemed pretty obvious but I knew there was something more to it. It has always torn me apart on the inside trying to prove that I knew I was fit. During this time I became very callused emotionally and really struggled with the fact that I couldn't push my body to its physical limits although I knew I had more in me. From this point on was the loneliest and toughest part of my life but it didn't stop me from putting in the work or trying to figure out a solution.

Starting my sophomore year, the pattern continued where I would run well early in the fall semester and trail off as the season progressed. Additionally, early during the fall of $2014 \mathrm{my}$ heartrate was very irregular and high which led to being diagnosed with Supraventricular 
Tachycardia (SVT) so I ended up having a cardiac ablation procedure. We had hoped this may have been a contributor to my breathing issues but shortly after I started running again I realized it was unrelated. I made it through the season racing slightly better than my freshman year until the outdoor track conference meet where I had such severe breathing attacks that my pace was reduced to a jog and I finished last in two races.

Naturally after years of hearing that l'm overtraining and I'm a "head-case", I started overanalyzing everything I was doing looking for a cause to my running issues. During the summer between my sophomore and junior year of college I came up with a brilliant solution- I would get so fit that I could overpower my breathing problems; unfortunately, that didn't work either.

Early during my junior season in 2015 I started having consistent throat pain, so I returned to the immunologist. She suggested I might have Vocal Cord Dysfunction (VCD) which seemed to fit after looking into it on my own. I had unexplained voice cracks during conversations that didn't align with puberty, I stuttered on and off which developed around the same time as my breathing issues got worse, and I developed a habit cough that would get worse with running at times. I worked with multiple Speech Pathologists and eventually ended up with a pathologist who worked specifically with athletes. Following a speech therapy session there were good and bad days of running still. I ended up being tested for GERD and tested positive. I was super excited for this diagnosis as well since GERD had been linked to causing VCD. Unfortunately, treating the GERD didn't seem to help my breathing attacks much.

During the summer of 2016 , I ran 100 miles a week for 10 weeks straight and was feeling okay. I was still having breathing attacks but I felt like I had some control over them as I was still using a POWERbreathe ${ }^{\circledR}$ device most of the time. Like the previous college years, the fall season started out great but went downhill fast. I started seeing a sports psychologist in the fall of 2016 who worked with an NBA team in the area as well but this also didn't seem to have any lasting effect. After the GERD treatment failed to have any real effect, I got a series of three Botox injections. At first, I felt like these injections helped but it was very short-lived and also had no lasting effect. During this time, I also started taking amitriptyline which also seemed to have very little effect on the breathing issues.

In the spring of 2017 I was referred to the National Jewish Hospital (NJH) in Denver, CO. Due to classes, I ended up scheduling a series of appointments over a ten-day period that summer. By this point the self-doubt and the inability to do what I had dreamed of being the best at were taking a toll on me. It was at this time where I started having breathing difficulties in everyday life. Stairs were harder to get up. I would often have to cut off a friend midconversation just to catch my breath after climbing just one flight. I couldn't talk on long runs anymore because I would mumble and slur my words. These speech difficulties got worse to where I would have difficulty just having conversation while walking long distances across campus. I would often be light-headed and dizzy after long easy runs; I couldn't wait until Denver.

During my ten-day stent in Denver that summer, I saw a myriad of doctors at NJH. Three inlet patches were found in my throat. I ended up having ablation therapy on the patches and it was thought that I would be back to running without issue in 2-3 months. In conjunction with the ablation therapy I was also put on Nexium which didn't help much either. There was one physician at NJH that was doing research on vocal cord disorders but he was on vacation during this trip so I scheduled to see him in December of 2017.

In the Fall of 2017, I started my last crosscountry season in college and I was mentally not into it. Before I knew it, I was back at NJH in 
December. I had one more ablation procedure on a small portion of one inlet patch and saw the physician who was researching vocal cord disorders (Dr. Olin). I went through a series of treatment sessions with Dr. Olin where I was taught how to breathe during exercise. To this day, this has been the single greatest help to my breathing.

The Spring of 2018 was a turning point in my life. I began to put life after college as a priority while continuing to run. Since then, I have only run a few 100-mile weeks while continually averaging 50 miles per week. There is no doubt that the breathing techniques I learned with Dr. Olin have helped. I still have breathing issues although they're not as frequent or severe. I continue to have heartburn, albeit less severe. If I miss a day of taking Nexium then I notice my breathing is worse.

\section{SUPPLEMENTAL VIDEOS}

Video 1: Example of Exercise-Induced Shortness of Breath

Video 2: Visualization of Airway Obstruction

Video 3: Continuous Laryngoscopy During Exercise

\section{REFERENCES}

1. Hull JH, Ansley L, Robson-Ansley P, Parsons JP. Managing respiratory problems in athletes. Clin Med (Lond). 201 2;1 2(4):351 356.

https://doi.org/10.7861/clinmedicine.12-4351.

2. Lund T, Pedersen L, Larsson B, Backer V. Prevalence of asthma-like symptoms, asthma and its treatment in elite athletes. Scand J Med Sci Sports. 2009;19(2):174-178. https://doi.org/10.1111/i.16000838.2007.00753.x.

3. Christensen PM, Thomsen SF, Rasmussen N, Backer V. Exercise-induced laryngeal obstructions: prevalence and symptoms in the general public. Eur Arch Otorhinolaryngol. $2011 ; 268(9): 1313-1319$. https://doi.org/10.1007/s00405-011 $1612-0$.

4. Maat RC, Roksund OD, Halvorsen T, et al. Audiovisual assessment of exercise-induced laryngeal obstruction: reliability and validity of observations. Eur Arch Otorhinolaryngol. 2009;266(12):1929-1936. https://doi.org/10.1007/s00405-0091030-8.

5. Nielsen EW, Hull JH, Backer V. High prevalence of exercise-induced laryngeal obstruction in athletes. Med Sci Sports Exerc. 2013;45(1 1 1):2030-2035. https://doi.org/10.1249/MSS.0b013e3182 98b19a.

6. Morris MJ, Christopher KL. Diagnostic criteria for the classification of vocal cord dysfunction. Chest. 2010;138(5):1 213 -1 223. https://doi.org/10.1378/chest.09-2944.

7. Hull JH, Hull PJ, Parsons JP, Dickinson JW, Ansley L. Approach to the diagnosis and management of suspected exercise-induced bronchoconstriction by primary care physicians. BMC Pulm Med. 2009;9:29. https://doi.org/10.1186/1471-2466-9-29.

8. Olin JT, Clary MS, Fan EM, et al. Continuous laryngoscopy quantitates laryngeal behaviour in exercise and recovery. Eur Respir J. 2016;48:1192-1200. https://doi.org/10.1183/13993003.00160 -2016 .

9. Silverman $M$, Anderson SD. Standardization of exercise tests in asthmatic children. Arch Dis Child. 1972;47(256):882-889. https://dx.doi.org/10.1 136\%2Fadc.47.256. 882.

10. Halvorsen T, Walsted ES, Bucca C, et al. Inducible laryngeal obstruction: an official joint European Respiratory Society and European Laryngological Society statement. Eur Respir J. 2017;50(3). https://doi.org/10.1183/13993003.02221 -2016 . 
11. Heimdal JH, Roksund OD, Halvorsen T, Skadberg BT, Olofsson J. Continuous laryngoscopy exercise test: A method for visualizing laryngeal dysfunction during exercise. Laryngoscope. Vol 1 162006:52-57. https://doi.org/10.1097/01.mlg.00001845 28.16229.ba.

12. Tervonen $H$, Niskanen $M M$, Sovijarvi $A R$, Hakulinen AS, Vilkman EA, Aaltonen LM. Fiberoptic videolaryngoscopy during bicycle ergometry: A diagnostic tool for exerciseinduced vocal cord dysfunction. Laryngoscope. 2009;1 19(9):1776-1780. https://doi.org/10.1002/lary.20558.

13. Olin JT, Clary MS, Connors D, et al. Glottic configuration in patients with exerciseinduced stridor: A new paradigm. Laryngoscope. 2014;1 24(1 1):2568-2573. https://doi.org/10.1002/lary.24812.

14. Olin JT, Deardorff EH, Fan EM, et al. Therapeutic laryngoscopy during exercise: $A$ novel non-surgical therapy for refractory EILO. Pediatr Pulmonol. 2017;52(6):813819. https://doi.org/10.1002/ppul.23634.

15. Johnston KL, Bradford $H$, Hodges $H$, Moore CM, Nauman E, Olin JT. The Olin EILOBI breathing techniques: Description and initial case series of novel respiratory retraining strategies for athletes with exercise-induced laryngeal obstruction. J Voice.

2018;32(6):698-704. https://doi.org/10.1016/i.jvoice.2017.08.0 20.

16. Graham S, Deardorff E, Johnston K, Olin JT. The fortuitous discovery of the olin eilobi breathing techniques: A case study. J Voice. 2018;32(6):695-697. https://doi.org/10.1016/i.jvoice.2017.08.0 19.

17. Altman KW, Mirza N, Ruiz C, Sataloff RT. Paradoxical vocal fold motion: presentation and treatment options. J Voice.

2000; 14(1):99-103. 